\title{
Compact Groups of Galaxies found using the COSMOS Catalogue
}

\author{
D. G. Barnes ${ }^{1}$ and M. J. Drinkwater \\ Anglo-Australian Observatory, Coonabarabran, NSW 2357, Australia
}

\begin{abstract}
.
We have developed an algorithm to search the COSMOS/UKST Southern Sky Object Catalogue for compact groups of galaxies, with the aim of finding regions of high mass-to-light ratio. With this catalogue, and quantitative criteria, we select groups objectively and therefore hope to improve upon earlier visual searches. We use a density enhancement measure to define a compact group.

We have made a preliminary search of 15 Schmidt fields (total area $\sim 390 \mathrm{deg}^{2}$ ), finding 58 compact groups, ie. a mean density of 0.15 compact groups $\operatorname{deg}^{-2}$. In this paper we present finding charts and images of some of the compact groups found.
\end{abstract}

\section{Introduction}

Compact groups of galaxies are poor but dense associations of galaxies. They appear as enhancements to the two-dimensional density of field galaxies on survey plates. Most of these associations are real physical systems and it is possible that they may trace regions of high mass-to-light ratio.

Rose (1977) studied the distribution of compact groups of galaxies on the Palomar Observatory Sky Survey (POSS) plates. He found that nearly every compact group had at least one galaxy nearer to it than would be expected from a random field distribution of galaxies. Rose concluded that compact groups are, in general, transient configurations of larger groups.

Hickson (1982) identified 100 compact groups by systematically searching the POSS red prints. His catalogue contains the densest systems of galaxies known, with space densities reaching four orders of magnitude higher than densities typical of the centres of rich clusters. Hickson \& Rood (1988) demonstrated that nearly all of the Hickson compact groups are true dynamical systems.

Both Rose and Hickson's studies were based on visual searches; in this paper we present preliminary results of a machine-based automated search for compact groups.

\footnotetext{
${ }^{1}$ Current address: Department of Physics, University of Melbourne, Parkville, Victoria 3052, Australia
} 


\section{The COSMOS/UKST Southern Sky Object Catalogue}

COSMOS is the photographic-plate scanning facility of the Royal Observatory, Edinburgh (ROE) which has been providing digitised scans of Schmidt plates for several years. From all the plates scanned, a digitised copy of the UKST Southern $J$ survey has been produced. In raw image form this represents a very large amount of data (about 1 GByte per plate), but this can be greatly reduced by storing the data as a list of the images detected, each parameterised by a few real numbers. This format is sufficient for most uses of the data, giving positions, magnitudes, classifications, moments and profiles. From this data pseudo-finding charts can be generated and selected classes of objects can be chosen automatically for various projects. We note that for reliable identification of individual objects, particularly near bright stars which may be mis-classified by COSMOS, direct visual inspection of the survey plates is essential.

In order to facilitate access to the data, ROE has collaborated with the Naval Research Laboratory in Washington D.C. to produce a compressed version of the image list, known as the COSMOS/UKST Southern Sky Object Catalogue (Yentis et al, 1992). The catalogue covers all fields with Dec $\leq 0^{\circ}$ excluding the most crowded Galactic Plane and Magellanic Cloud regions down to a limiting magnitude of $B_{J}=22.5$. This version is 6 GByte in size and has been installed at the Anglo-Australian Observatory. We have written software to enable fast access to any part of the catalogue making statistical projects like the present search very efficient.

\section{Objective search criteria}

We have searched the COSMOS catalogue for compact groups of galaxies that satisfy the following quantitative definition, modelled on Rose's (1977) criteria:

- We examine collections of $n$ galaxies $(3 \leq n \leq 7)$ with $B_{J}$ magnitudes in the range 12.5-17.5. We define an area $A$ for the group as that of the smallest circle enclosing the $n$ galaxies, centred on the galaxy from which the sum of the squared distances to the other $(n-1)$ galaxies is a minimum. We denote by $\sigma$ the local number density of galaxies with such magnitudes, the average being taken over a large $\left(\sim 1^{\circ} \times 1^{\circ}\right)$ region of sky surrounding the galaxies.

- We then define a density enhancement,

$$
\rho=\frac{n}{A \sigma} \text {. }
$$

- When $\rho>70$ we consider the collection of galaxies to be a compact group. ( $A \sigma$ is not strictly the expected number of galaxies in the area $A$ because there is always one galaxy at the centre of the area in consequence of our search method.) 


\section{Compact groups detected}

We searched 15 Schmidt fields in a region around the South Galactic Pole. 85 groupings of galaxies satisfied the above criteria. After visually checking each candidate group, and eliminating known Abell Clusters (our definition has no isolation condition), we accepted 58 compact groups. Table 1 gives a summary of the search results.

Table 1. Compact group detections in $\sim 390 \mathrm{deg}^{2}$.

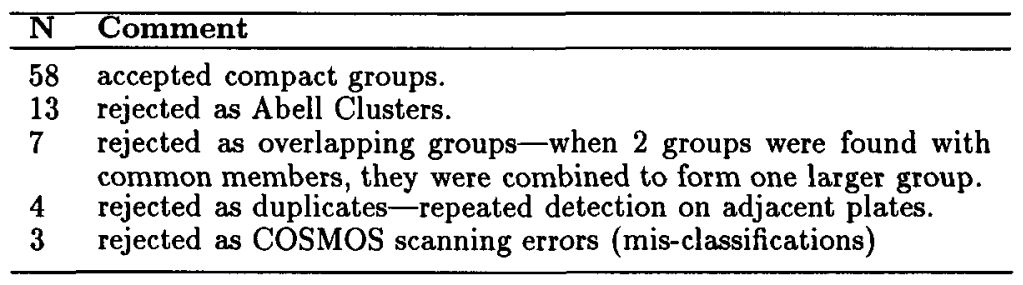

85 Total detected.

The mean density of accepted compact groups in our survey is 0.15 per square degree. Rose (1977) detected 0.07 (3-member) groups per square degree. These numbers cannot be directly compared, since we have calculated background density, enhancement and group area in a slightly different manner to Rose. Nevertheless, we obtain a detection rate of the same order as Rose. The important difference though is that our search is based on digitised data analysed automatically.

Table 2 summarises the classification of the 58 compact groups. The table shows the number of groups found with a certain population, and exhibiting particular density enhancements. Our proportion of groups with more than 3 members is significantly higher than that of Rose, we expect this is again due to our changes to Rose's definition.

Table 2. Compact group populations and density enhancements.

\begin{tabular}{ccccccc}
\hline \multirow{2}{*}{ Enhancement } & 3 & 4 & 5 & 6 & Total & Density $\left(\mathrm{deg}^{-2}\right)$ \\
\hline$>70$ & 46 & 6 & 5 & 1 & 58 & 0.15 \\
$>100$ & 31 & 3 & 1 & 0 & 35 & 0.09 \\
$>200$ & 15 & 2 & 1 & 0 & 18 & 0.05 \\
\hline
\end{tabular}

Two examples of detected compact groups are given in Figs 1 and 2. These show photographic enlargements of the survey plates and corresponding COSMOS finding charts. Table 3 lists the position, number of members, and approximate density enhancement for these two groups. 


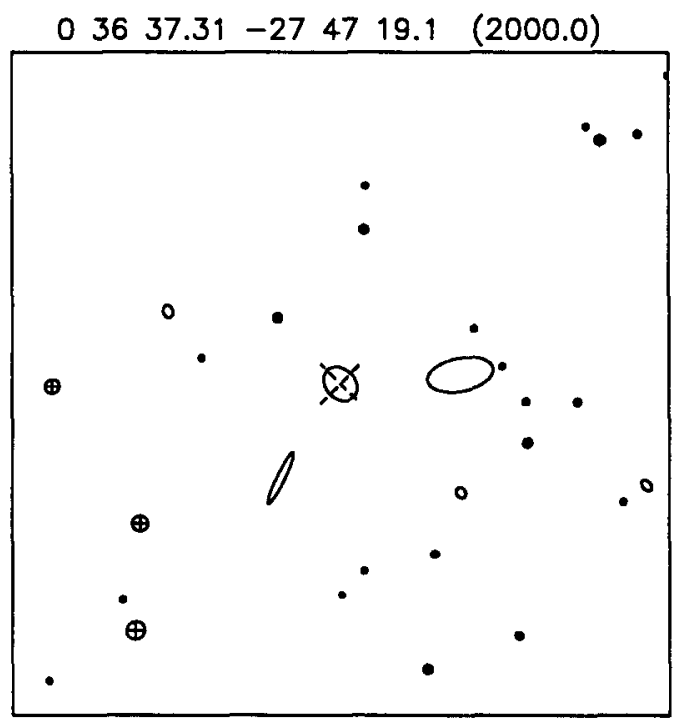

1 field $0411\left(12.0^{\prime} \times 12.0^{\prime}\right)$

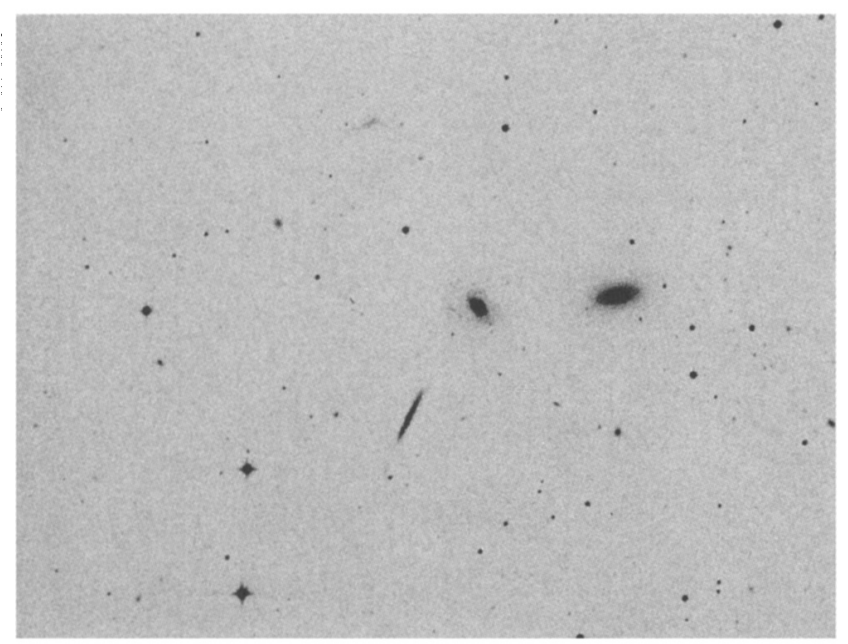

Figure 1. J survey image and COSMOS finding chart (limited to $\left.B_{J}<18.5\right)$ for compact group $00 \mathrm{~h} 37 \mathrm{~m}-27^{\circ} 47^{\prime}$. NE is at the top left. 

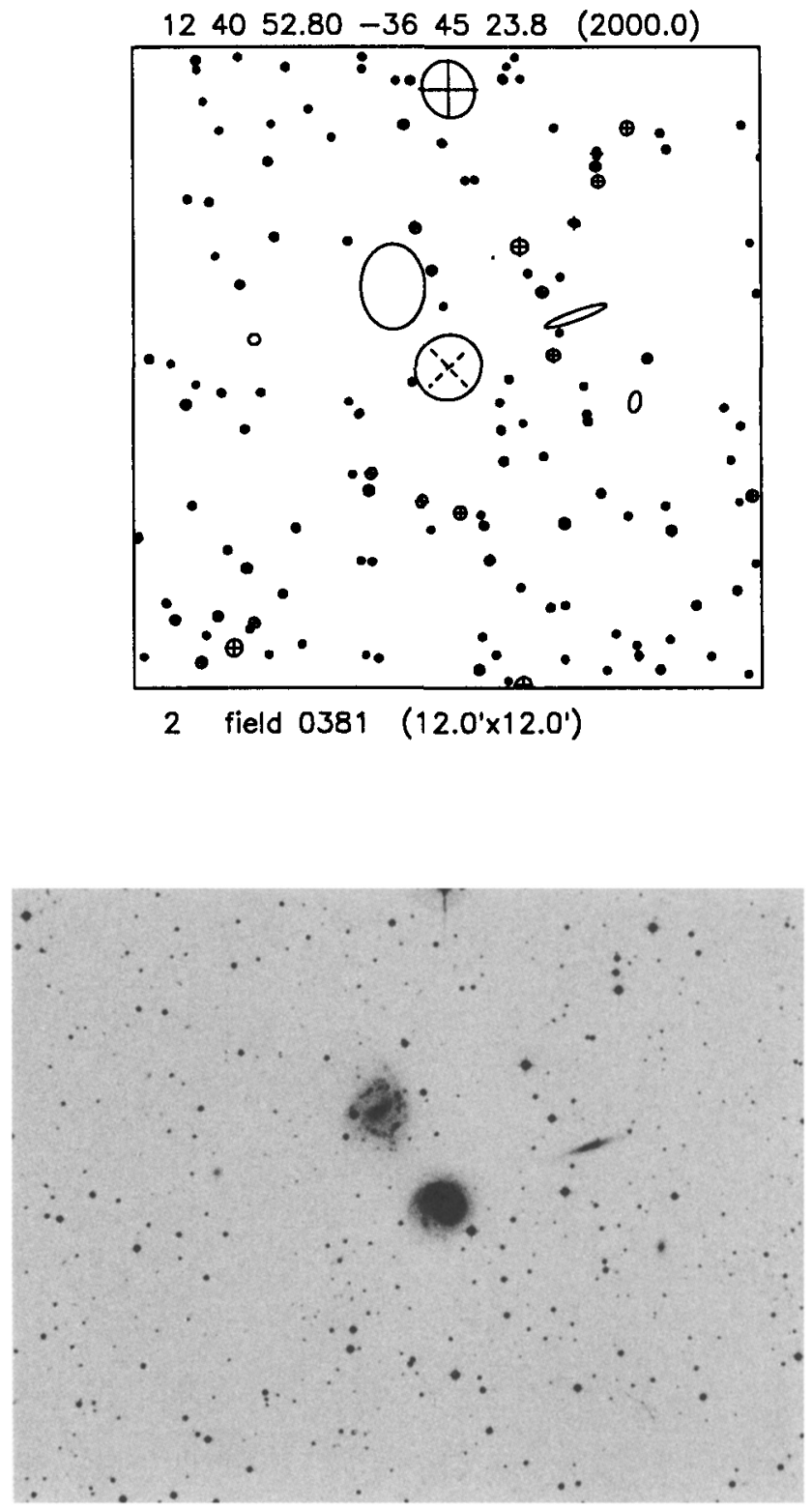

Figure 2. Compact group $12 \mathrm{~h} 41 \mathrm{~m}-36^{\circ} 45^{\prime}$ 
Table 3. Properties of two example compact groups.

\begin{tabular}{ccccc}
\hline Figure & RA $(2000)$ & Dec $(2000)$ & Members & Enhancement \\
\hline 1 & $00^{h} 37^{m}$ & $-27^{\circ} 47^{\prime}$ & 3 & 140 \\
2 & $12^{h} 41^{m}$ & $-36^{\circ} 44^{\prime}$ & 3 & 75 \\
\hline
\end{tabular}

Note: Group 4 was found in a part of the sky separate to the main region that we searched.

\section{Velocities and distances-two confirmed groups}

Not all two-dimensional groups are physical-indeed a Monte Carlo simulation of our survey with randomised $x$ - and $y$-coordinates suggested that up to $20 \%$ of the associations could be chance projections. In order to confirm that a particular group is in fact a dynamical system, we need to obtain redshifts of the members to show that they all lie at roughly the same distance.

We have obtained a small number of velocities for the galaxies of two compact groups from 2 fields near $\mathrm{RA}=12^{h}$, using the Australian National University 2.3 metre telescope at Siding Spring Observatory. Although our data are not complete enough to determine mass-to-light ratios, we have confirmed for both groups that the member galaxies lie at similar distances, and are therefore dynamically associated. Table 4 lists the positions and velocities (where available) of the galaxies of these two groups.

Table 4. Radial velocity data

\begin{tabular}{ccl}
\hline RA (2000) & Dec (2000) & Velocity(heliocentric) (km/s) \\
\hline $12^{h} 40^{m} 40.78^{s}$ & $-36^{\circ} 44^{\prime} 22.5^{\prime \prime}$ & $3210 \pm 60$ \\
$12^{h} 40^{m} 58.18^{s}$ & $-36^{\circ} 43^{\prime} 55.0^{\prime \prime}$ & $3600 \pm 100$ \\
$12^{h} 40^{m} 52.80^{s}$ & $-36^{\circ} 45^{\prime} 23.8^{\prime \prime}$ & $3747 \pm 100$ (de Grijp et al. 1992) \\
$11^{h} 45^{m} 19.33^{s}$ & $-44^{\circ} 25^{\prime} 15.1^{\prime \prime}$ & \\
$11^{h} 45^{m} 24.06^{s}$ & $-44^{2} 25^{\prime} 26.7^{\prime \prime}$ & $9810 \pm 100$ \\
$11^{h} 45^{m} 18.40^{s}$ & $-44^{\circ} 26^{\prime} 42.2^{\prime \prime}$ & $10490 \pm 60$ \\
$11^{h} 45^{m} 47.02^{s}$ & $-44^{\circ} 24^{\prime} 41.8^{\prime \prime}$ & $10320 \pm 200$ \\
\hline
\end{tabular}

\section{Conclusions}

We have performed a machine-based, automated, objective search of a digital sky catalogue, for compact groups of galaxies. We found 58 compact groups in $390 \mathrm{deg}^{2}$; a mean density of $0.15 \mathrm{deg}^{-2}$. Given a suitable algorithm and definition, our method could be adapted to search for almost any kind of star or galaxy association. In the future we hope to extend our search to produce a larger sample of compact groups of galaxies. A complete redshift survey of the 
galaxies of those groups would indicate which of the groups are true dynamical systems, and enable the calculation of mass-to-light ratios for a large sample of compact groups. We could then study the existence and form of dark matter in small, dense systems of galaxies.

Acknowledgements. DGB would like to thank the Anglo-Australian Observatory for a summer vacation scholarship. We acknowledge the use of the COSMOS/UKST Southern Sky Object Catalogue as supplied by the AngloAustralian Observatory. This research has made use of the NASA/IPAC Extragalactic Database (NED) which is operated by the Jet Propulsion Laboratory, Caltech, under contract with the National Aeronautics and Space Administration.

\section{References}

de Grijp M. H. K., Keel W. C., Miley G. K., Goudfrooij P. \& Lub J., 1992, A\&AS, 96, 389

Hickson P., 1982, ApJ, 255, 382

Hickson P. \& Rood H. J., 1988, ApJ, 331, L69

Rose J. A., 1977, ApJ, 211, 311

Yentis D.J., Cruddace R.G., Gursky H., Stuart B.V., Wallin J.F., MacGillivray H.T. \& Collins C.A., 1992, in Digitised Optical Sky Surveys, H.T. MacGillivray \& E.B. Thomson, eds, (Kluwer, Dordrecht), p. 67 